\title{
Avaliação psicométrica de três questionários sobre o historial familiar
}

\section{Evaluation of psychometric properties of three questionnaires addressing early experiences in family context}

\author{
Ivandro Soares Monteiro ${ }^{1}$, Angela da Costa Maia ${ }^{2}$ \\ 1 Psicólogo e assistente no Instituto Superior da Maia, Maia, Portugal. \\ 2 Psicóloga e professora auxiliar na Universidade do Minho, Braga, Portugal.
}

Recebido: 30/3/2009 - Aceito: 9/9/2009

\begin{abstract}
Resumo
Contexto: A família é a arena primária na qual a criança desenvolve a sua sociabilização. As experiências tidas neste sistema podem constituir um factor protector ou de risco, contribuindo para a sua vulnerabilidade ou resiliência diante de desordens mentais quando adulto. A avaliação da história familiar de experiências emocionais relevantes é problemática, e o uso de instrumentos psicométricos pode auxiliar a superar certas limitações metodológicas. Objectivos: Desenvolver três subquestionários para avaliação das experiências emocionais decorridas no contexto familiar da infância até a adolescência. Esses instrumentos são adaptações para o português do Family Background Questionnaire (FBQ) tratando dos cuidados paliativos e do ambiente familiar e são administrados retrospectivamente a adultos. Métodos: Três versões do FBQ que tratam de (1) cuidados paternos, (2) cuidados maternos e (3) ambiente familiar foram produzidas. Os questionários foram aplicados a 280 participantes. A avaliação das propriedades psicométricas desses questionários teve como base a determinação da consistência interna, análise factorial e correlação de Spearman. Resultados: Os três questionários mostraram-se adequados para avaliação de seus respectivos domínios. Conclusões: A avaliação objetiva das primeiras experiências emocionais no contexto familiar produz insights importantes para a compreensão do desenvolvimento psicológico, com implicações clínicas e de investigação.
\end{abstract}

Monteiro IS, Maia AC / Rev Psiq Clín. 2010;37(3):97-104

Palavras-chave: Desenvolvimento, família, experiências, cuidados, questionários.

\begin{abstract}
Background: Family is the primary setting where children develop their social skills. Early experiences may represent protective or risk factors that interact with their vulnerability to or resilience against mental disorders in adulthood. The assessment of the family history of relevant emotional experiences is problematic, and the use of psychometric instruments may help overcome certain methodological limitations. Objectives: To develop three sub-questionnaires for the assessment of early emotional experiences in the family context in childhood through adolescence. These instruments are Portuguese adaptations of the Family Background Questionnaire (FBQ), addressing aspects of parental care and family environment, and are retrospectively administered to adults. Methods: Three composite versions of the FBQ addressing (1) Paternal care, (2) Maternal care and (3) Family environment were produced. Questionnaires were administered to 280 participants. The assessment of the psychometric properties of these questionnaires was based on the determination of internal consistency, factor analysis and Spearman's correlations. Results: All three questionnaires proved adequate for the assessment of their respective domains. Discussion: The objective assessment of early emotional experiences in the family context yields important insights to the comprehension of psychological development, with both clinical and research implications.
\end{abstract}

Monteiro IS, Maia AC / Rev Psiq Clín. 2010;37(3):97-104

Keywords: Development, family, experiences, cares, questionnaires.

\section{Introdução}

Sabemos que as características da família de cada indivíduo têm uma influência significativa no seu desenvolvimento psicossocial, com implicação quer na infância, quer ao longo da vida. Há também um consenso evidente no que concerne ao facto de que a família é a "arena primária" onde a criança vai desenvolver a sua socialização. A família e as experiências familiares podem constituir um factor protector ou de risco, mediante a sua capacidade de assegurar ou não protecção, sobrevivência e bem-estar da criança ${ }^{2}$. O desenvolvimento da criança e o seu bem-estar psicológico posterior podem ser afectados pelas situações de adversidade no ambiente familiar ${ }^{3}$. Nessa perspectiva desenvolvimental, quanto mais cedo for a exposição à violência ou a formas de cuidado inadequadas, mais consequências negativas no desenvolvimento são esperadas ${ }^{4}$, pois a regulação dos padrões emocionais tende a ser estruturada durante a infância e adolescência ${ }^{5}$. São vários os estudos que evidenciam uma influência directa das vivências experienciadas na família de origem sobre indicadores de saúde na vida adulta. Por exemplo, o estudo das Experiências Adversas na Infância (do inglês ACE - Adverse Childhood Experiences) é um ambicioso projecto de investigação epidemiológico americano que estuda, em larga escala, a forma como as experiências de infância afectam a saúde na vida adulta, décadas mais tarde ${ }^{6}$. Esse grupo de investigação considera dez categorias de experiências adversas durante a infância, consideradas até os 18 anos, três das quais constituem abuso pessoal recorrente - 1) abuso físico recorrente, 2) abuso emocional recorrente ou 3) abuso sexual; cinco que constituem uma disfunção familiar, a saber: 4) a existência de um abusador de álcool ou drogas; 5) um membro da família nuclear encarcerado (prisioneiro); 6) alguém que sofra de depressão crónica, doença mental ou tenha comportamentos suicidas; 7) violência doméstica (mãe agredida violentamente); 8) um ou nenhum pai (separação, divórcio ou falecimento dos pais); e duas que avaliam a negligência, a saber: 9) Negligência física; 10) Negligência emocional. O ACE tem desenvolvido vários estudos que evidenciam uma relação clara entre adversidades vividas durante a infância e a diminuição da qualidade de vida ou desenvolvimento 
de perturbações físicas e psicológicas durante a idade adulta ${ }^{7,8}$. Podemos referir outros estudos que mostram uma relação clara entre experiências infantis e saúde mental na idade adulta ${ }^{9,10}$.

O estudo do impacto das experiências adversas no desenvolvimento tem evidenciado o papel preponderante da responsividade e aceitação parental, pelo que as diferentes perspectivas têm formado a base de várias considerações teóricas sobre a relação entre a qualidade da relação familiar e o desenvolvimento pessoal11,12.

No extremo dos cuidados inadequados estão os maus-tratos. Nos Estados Unidos da América, o Departamento de Saúde e Serviços Humanos desenvolveu uma vasta categorização de diferentes formas de maus-tratos infantis (por exemplo, abuso emocional, negligência emocional, abuso físico, negligência física, abuso sexual e negligência educacional), que pode ser usada na clarificação da natureza e impacto da ausência de cuidados parentais adequados ${ }^{13}$. É amplamente aceite que essas formas de abuso têm um impacto significativo no desenvolvimento infantil, e a intensidade, duração e interacção entre as várias formas de maus-tratos parecem ser factores mediadores dos seus efeitos ${ }^{14,15}$. Daqui se deduz que conhecer a história familiar e de desenvolvimento é crucial para uma melhor compreensão das experiências e vivências do adulto, assim como para uma mais eficaz avaliação dos seus recursos, resiliências e vulnerabilidades.

Daí que o desenvolvimento de instrumentos que permitam avaliar de forma objectiva essas características tenha sido realizado ao longo dos anos, por forma a evidenciar essas vivências familiares.

O método mais usado é o da entrevista, quer na clínica, quer na investigação. Contudo, são vários os instrumentos alternativos. Numa revisão da literatura realizada há quase 20 anos $^{1}$, encontraram-se trinta e dois instrumentos construídos para medir memórias de adultos de, pelo menos, uma característica da sua família de origem.

Essa revisão ${ }^{1}$ evidenciou que a maioria dos instrumentos avaliou um número limitado de variáveis, e mesmo os mais compreensivos não avaliavam os factores que normalmente são incluídos na avaliação clínica da história da família de origem. De facto, nenhum deles avaliava simultaneamente factores, tais como incesto, negligência física, abuso parental de substâncias, desajustamento psicológico parental, estressores familiares ou mecanismos de coping.

Para além dessa limitação de variáveis, verificaram-se outros problemas ${ }^{1}$ : muitos dos instrumentos de avaliação da família de origem incluem questões pouco específicas sobre comportamentos ou que averiguam sobre sentimentos que a pessoa tinha quando era criança ou então faz julgamentos sobre algum membro familiar (por exempo, Parental Bonding Instrument ${ }^{16}$ ). O autor dá exemplos de itens como "os meus pais não queriam que eu crescesse", ou "os meus pais eram extremamente protectores", que fazem com que as pessoas com fraco insight sobre o comportamento dos seus pais tenham grandes dificuldades de os julgar. Segundo o mesmo autor, é difícil interpretar respostas para esses itens de forma isolada, sem outras informações comportamentais ou mais objectivas que permitam contextualizar os sentimentos que estão a ser expressos.

Melchert ${ }^{1}$ salientou ainda o facto de esses instrumentos avaliarem as características da família de origem de uma forma global, não permitindo, a sua grande maioria, uma avaliação separada sobre diferenças que são importantes perceber, como, por exemplo, o comportamento do pai e da mãe. Atendendo às limitações que foram identificadas nos instrumentos disponíveis, esse autor sentiu a necessidade de desenvolver um novo instrumento que permitisse avaliar, de forma compreensiva, comportamental e específica, as características da família de origem. É com base neste trabalho do Family Background Questionnaire, desenvolvido nos Estados Unidos da América, por Melchert ${ }^{1,17}$, que nos propusemos desenvolver formas de avaliação dos cuidados familiares para a população portuguesa jovem.

Esta foi a primeira vez que o Family Background Questionnaire (FBQ1) foi trabalhado para uma língua estrangeira, nomeadamente o português. Ao fazê-lo, e dadas as diferenças culturais, várias questões da versão original tiveram de ser alteradas. O presente artigo apresenta os procedimentos de desenvolvimento de três questionários independentes, que foram concretizados a partir do FBQ, de modo a obter o Questionário dos Cuidados Paternos, Questionário dos Cuidados Maternos e o Questionário do Ambiente Familiar.

A nossa opção pelo original $\mathrm{FBQ}^{1}$ teve como objectivo obter um material em português para obtenção de informação sobre experiências decorridas durante a infância no contexto familiar de uma forma detalhada, constituindo-se como um estudo prévio para a realização de três estudos sobre a relação entre as experiências familiares no desenvolvimento e a psicopatologia depressiva na idade adulta ${ }^{18}$. Este artigo tem como finalidade apresentar os procedimentos que foram realizados num estudo de adaptação à população portuguesa, bem como os dados psicométricos da validação do FBQ, incluindo três escalas. Uma tem como objectivo a avaliação dos cuidados paternos, a segunda avalia cuidados maternos e a terceira avalia o ambiente familiar experienciado durante a infância e adolescência, sendo dirigidas para indivíduos adultos.

\section{Método}

\section{Participantes}

O presente estudo foi realizado com um total de 280 participantes, estudantes de uma instituição do ensino superior privado, no norte de Portugal, de vários cursos superiores (gestão, psicologia, educação física, multimédia, solicitadoria e aconselhamento), 145 do sexo feminino $(51,80 \%)$ e 135 do sexo masculino $(48,20 \%)$. As idades estão compreendidas entre os 18 e os 57 anos $(\mathrm{M}=21,07$ anos, DP $=4,66$ anos). Quanto ao estado civil, 93,20\% são solteiros $(\mathrm{n}=261)$, $3,2 \%$ são casados $(n=9), 2,1 \%$ são unidos de facto $(n=6), 1,1 \%$ é divorciado $(n=3)$ e $0,4 \%$, viúvo $(n=1)$.

\section{Instrumento}

$\mathrm{O}$ original $\mathrm{FBQ}^{1}$ é constituído por 179 itens, distribuídos por 22 subescalas. A sua cotação dá origem a um valor global do funcionamento da família de origem, que é calculado pela junção de vários itens de quase todas as subescalas (excepto as subescalas de tarefas domésticas, abuso sexual e abuso físico do pai e da mãe, cujos itens não entram para a pontuação do nível de funcionamento familiar, pois funcionam como checklists na versão original).

A aplicação é dirigida a adultos e faz questões sobre as suas memórias de experiências na família de origem até os 18 anos de idade. Uma escala tipo lickert de um a cinco pontos é usada para todos os itens do questionário. Todos as pontuações são cotadas de forma a que as mais elevadas indiquem melhor nível de funcionamento familiar. Assim, no geral, varia num continuum de 1 a 5 , em quanto mais próximo de 5 melhor funcionamento familiar. Relativamente a checklists, são constituídas por questões qualitativas do tipo "sim" e "não".

As 22 subescalas que constituem esse instrumento na versão americana são as seguintes: Responsividade do Pai e Responsividade da Mãe (versus Negligência Educacional), Aceitação do Pai, Aceitação da Mãe (versus Abuso Emocional), Abuso Físico do Pai e Abuso da Mãe, Envolvimento Educacional do Pai e Envolvimento Educacional da Mãe, Estilo de Tomada de Decisão do Pai e Estilo de Tomada de Decisão da Mãe, Abuso de Substâncias do Pai e Abuso de Substâncias da Mãe, Ajustamento Psicológico do Pai e Ajustamento Psicológico da Mãe, Abuso Sexual, Negligência Física, Controlo Parental, Participação em Tarefas Domésticas, Expressão Afectiva, Coligação Parental, Apoio Social e Estressores Familiares. As subescalas de Abuso Sexual - 5 itens - e Abuso Físico do Pai - 2 itens - e da Mãe - 2 itens - funcionam como checklists, pelo que não são usadas para o cálculo de qualquer escala.

Para facilitar a compreensão conceptual das escalas, apresentamos uma breve descrição delas ${ }^{1,17}$. A responsividade parental, que se refere à capacidade de resposta no momento certo às necessidades dos filhos, e a aceitação (aceitar as atitudes e opiniões dos filhos) podem ser consideradas como um continuum que vai desde "caloroso, cuidadoso, de confiança, com empatia responsiva" até "negligência de cuidados, rejeição e abuso". O Envolvimento Educacional é uma prática parental direccionada ao comportamento de estudo dos filhos, 
que engloba actividades como a participação em reuniões escolares, apoio nos trabalhos de casa, pesquisa e investigação para trabalhos e facilitação para participação em visitas de estudo. O Estilo de Tomada de Decisão é o conjunto de atitudes dos pais para com os seus filhos, a partir dos quais se cria o ambiente psicológico-emocional da relação entre ambos, é caracterizado por comportamentos que incluem as práticas parentais (elogios, gritos, punições etc.) e outros aspectos relacionais, tais como: tom de voz, linguagem corporal, descuido, atenção, mudanças de humor etc.; o Ajustamento Psicológico referese à saúde mental dos pais, nomeadamente perturbações de humor ou consumo de substâncias; o Abuso Sexual é todo acto ou jogo sexual cujo agressor está numa fase de desenvolvimento psicossexual mais adiantado que a vítima, desde actos em que não existe contacto físico (como, por exemplo, voyeurismo, exibicionismo) até diferentes outros com ou sem penetração (sexo vaginal, oral, anal, manipulação de genitália do agressor ou da vítima etc.); a Negligência Física é a falta de cuidados físicos (alimentação, vestuário, condições de higiene etc.); o Controlo Parental é o grau em que o sistema familiar é flexível no seu modelo educativo, desde o autocrático até ao democrático; a Participação em Tarefas Domésticas refere-se o grau de exploração da criança para as limpezas e cuidados domésticos (limpar o pó, cuidar de um irmão, aspirar etc.); a Expressão Afectiva caracteriza-se pela expressão visível de sentimentos positivos e negativos, com opiniões e demonstrações de carinho, e diferencia-se da responsividade na medida em que a expressão afectiva pode não ser dada no momento certo, o que evidencia que os pais podem ter expressão afectiva mas sem uma responsividade adequada. A Coligação Parental é o grau de acordo entre o pai e a mãe em relação às regras ou instruções aos filhos. O Apoio Social infantil refere-se à forma como a criança podia estar com amigos, conviver, ir ao cinema, estudar fora de casa etc.; e Estressores Familiares (problemas financeiros, discussões sérias familiares, um membro familiar encarcerado etc.).

Os dados psicométricos da versão original ${ }^{1}$ numa população não clínica, resultantes de um estudo com 517 sujeitos, evidenciam uma elevada consistência interna, não apresentando nenhuma das subescalas um alfa abaixo de, 76 .

\section{Procedimento}

Traduziu-se, com autorização do autor, o questionário americano ${ }^{1,13}$ mantendo o formato dos itens e das instruções. Para obter a tradução portuguesa, dois psicólogos, com experiência clínica de mais de cinco anos e bom conhecimento da língua inglesa, trabalharam até um consenso no que se refere à adaptação do texto da versão original à população portuguesa, para cada um das questões das escalas. Quando se tratava de expressões idiomáticas, mais do que uma tradução à letra das várias palavras, fez-se um esforço no sentido de captar o sentido conotativo da expressão original ${ }^{19}$, e por traduzi-la por uma expressão que, na língua portuguesa, pudesse ter o mesmo significado. Tentou-se, sempre que possível, traduzir os itens da forma mais directa possível, mas, sempre que não foi possível uma tradução literal, tentámos manter o sentido essencial do item original. Foram tidas em atenção as indicações de Van de Vijver e Hambleton ${ }^{20}$, que chamam a atenção para o facto de ser necessário assegurar que o processo de tradução/adaptação tenha em consideração as diferenças linguísticas e culturais da população para a qual se destina a versão traduzida e adaptada do instrumento. Uma outra técnica usada para avaliar a adequação da tradução de um teste psicológico é a da retroversão ${ }^{19,20}$. Assim, depois foi novamente traduzido para o inglês por uma pessoa bilíngue, a viver nos Estados Unidos da América. Todas as dúvidas tidas no desenvolvimento dos três questionários portugueses em relação à base do material original foram sempre esclarecidas com os autores do FBQ ${ }^{13}$.

A partir do momento em que os 179 itens estavam traduzidos, foram eliminados dois itens ("com que regularidade o seu pai o ajudava a escolher as turmas ou grupos de escola?") (“com que regularidade a sua mãe a ajudava a escolher as turmas ou grupos de escola?") porque não se aplicam na população portuguesa, por decisão consensual dos juízes. Em relação às perguntas restantes e sua classificação por parte do sujeito, não se fizeram praticamente alterações no que concerne ao conteúdo ou estrutura, dado que a tradução foi para uma linguagem o mais simplificada possível, de forma a que fosse facilmente compreendida pela maioria das pessoas, independentemente do seu grau de escolaridade ou cultural. Os seis itens relacionados com o abuso sexual durante a infância constituem uma checklist, pelo que não são contabilizados para o cálculo de qualquer subescala ou questionário. Mantivemos essa checklist devido à sua utilidade clínica.

Assim, após essa análise conceptual e atendendo à extensão do instrumento e conteúdo dos itens, os 179 itens ficaram reduzidos a 171 itens, que organizamos em três questionários diferentes: um para avaliar os cuidados paternos, outro para avaliar os cuidados maternos e o terceiro para avaliar o ambiente familiar. Dessa forma, aqueles itens que se referiam especificamente à relação com a mãe ou com o pai, tal como, por exemplo, "a minha mãe e o meu pai davam-me apoio e conforto sempre que eu precisava”, e ao qual era necessário responder separadamente para o pai e para a mãe, passou a ser um item no Questionário dos Cuidados Maternos (por exemplo, “ $a$ minha mãe dava-me apoio e conforto sempre que eu precisava") e outro item no Questionário de Cuidados Paternos (por exemplo, "o meu pai dava-me apoio e conforto sempre que eu precisava"). Os itens restantes, os quais se relacionavam com a qualidade do ambiente familiar, tais como acontecimentos familiares, a saúde mental dos pais, consumo de drogas ou de álcool, entre outros, foram inseridos no Questionário de Ambiente Familiar. Os 171 itens foram organizados nos três questionários, ficando inicialmente o Questionário de Cuidados Paternos e o Questionário de Cuidados Maternos constituídos com 45 itens cada um. O Questionário do Ambiente Familiar ficou, de início, constituído por 81 itens.

Cumprindo as normas éticas da instituição, e após a necessária autorização do Presidente do Conselho Directivo da instituição do ensino superior privado (com vários cursos na área da gestão, ciências sociais, desporto e tecnologia) e a obtenção do consentimento informado de cada um dos participantes dos vários cursos da instituição que voluntariamente aceitaram participar no estudo (em turmas aleatoriamente seleccionadas), os instrumentos foram aplicados em salas de aula, de forma colectiva. Os participantes foram também informados que existia a disponibilidade para proceder ao acompanhamento psicológico dos participantes afectados pelo questionário, em caso de necessidade, dado o teor deste, assim como foram ainda informados da possibilidade de desistir da participação em qualquer momento. A aplicação do material demorou cerca de 45 minutos, estando sempre presente um investigador.

Os dados para avaliação psicométrica foram todos introduzidos, analisados e trabalhados no programa estatístico SPSS (Statistical Package for Social Sciences - versão 16). Para a análise da validade de constructo, utilizaram-se os métodos de análise factorial, teste Kaiser-Meyer-Olkin e o teste de esfericidade de Bartlett e, para a análise da consistência interna, utilizaram-se as correlações item-total e o cálculo do alfa de Chronbach ${ }^{21}$. Para o cálculo das correlações entre os três questionários, foi usada a correlação de Spearman, após análise do teste de Kolmogorov-Smirnov ${ }^{21}$.

\section{Resultados}

Inicialmente, com vista a averiguar se seria ainda possível reduzir o número de itens, analisamos as correlações inter-item dentro de cada um dos três questionários, retirando, entre aqueles com correlação igual ou maior a ,80, o item menos claro em termos de compreensão da língua portuguesa, por decisão consensual dos juízes. Esse procedimento permitiu retirar da análise dois itens no questionário do pai (20 e 124), dois itens no questionário da mãe (21 e 125) e três itens no questionário do ambiente familiar $(67,95,154)$.

Um segundo critério para reduzir o número de itens foi por meio da análise loading de cada item constituinte em cada factor na análise factorial de cada um dos três questionários, cujo procedimento está embaixo descrito para cada um dos três questionários analisados. 


\section{Validade de constructo}

Para a validação de cada um dos três questionários, foi realizada a análise factorial dos componentes principais. Estabeleceu-se como critério mínimo ${ }^{21}$ de loading significativo para cada factor o valor de, 30 .

\section{a) Questionário de Cuidados Paternos}

Após uma primeira análise factorial com rotação varimax dos 44 itens constituintes desse questionário e sem forçar a nenhum factor, verificámos uma distribuição desses itens por oito factores. No entanto, sua distribuição não se agrupava em torno de nenhum constructo teórico bem definido e alguns desses factores tinham muito poucos itens. Com a análise do scree plot, percebeu-se que os dados eram compatíveis com a escolha de quatro factores. Com uma nova análise factorial forçada a quatro factores, os itens 38,40 , 48, 54 e 130 foram eliminados (devido aos loadings não atingirem o critério ,30 definido). Apesar de alguns itens saturarem em mais do que um factor, optámos pelo maior valor de loading e/ou pelo factor que melhor justificava a inclusão do item, ficando o Questionário de Cuidados Paternos constituído por 37 itens. O valor de KaiserMeyer-Olkin obtido foi de 95 , o que é considerado excelente ${ }^{21}$, e o teste de esfericidade de Bartlett é de $\mathrm{X}^{2}=6324.73$, o qual atinge um valor significativo $(\mathrm{p}<, 05)$, pelo que se rejeita a hipótese nula, ou seja, as variáveis são correlacionáveis. Nessa análise factorial, dos itens do Questionário de Cuidados Paternos, $41,10 \%$ da variância foi explicada pelo factor $1,6,86 \%$, pelo factor $2,4,35 \%$, pelo factor 3 e $4,02 \%$, pelo factor 4 . Os quatro factores explicaram a variância em $56,76 \%$, cujo resultado está dentro dos limites científicos aceitáveis ${ }^{21}$. Para verificar a forma como os itens se distribuem pelos quatro factores, consulte a tabela 1.

\section{b) Questionário de Cuidados Maternos}

Numa primeira análise factorial com rotação varimax dos 44 itens deste questionário sem forçar a nenhum factor, constatou-se uma distribuição por 10 factores. No entanto, sua distribuição não se agrupava em torno de nenhum constructo teórico bem definido e

Tabela 1. Loadings dos questionários de cada um dos pais na análise factorial forçada a quatro factores

\begin{tabular}{|c|c|c|c|c|c|c|c|c|c|}
\hline \multicolumn{5}{|c|}{ QUESTIONÁRIO DOS CUIDADOS PATERNOS } & \multicolumn{5}{|c|}{ QUESTIONÁRIO DOS CUIDADOS MATERNOS } \\
\hline Item & Factor 1 & Factor 2 & Factor 3 & Factor 4 & Item & Factor 1 & Factor 2 & Factor 3 & Factor 4 \\
\hline FB01 & 0,69 & & & & FB02 & 0,66 & 0,32 & & \\
\hline FB03 & 0,67 & 0,32 & & & FBO4 & 0,68 & 0,33 & & \\
\hline FB05 & 0,67 & & & & FBO6 & 0,64 & & & \\
\hline FB07 & 0,59 & 0,35 & & 0,36 & FB08 & 0,53 & 0,39 & & \\
\hline FB09 & 0,49 & & & 0,46 & FB010 & 0,49 & & & 0,36 \\
\hline FB011 & 0,66 & & & & FB012 & 0,72 & & & \\
\hline FB016 & 0,58 & & & & FB017 & 0,65 & & & \\
\hline FB018 & 0,73 & & & & FB019 & 0,64 & & & \\
\hline FB022 & 0,62 & & & & FB023 & 0,58 & & 0,40 & \\
\hline FB024 & 0,70 & & & & FB025 & 0,61 & & 0,33 & \\
\hline FBO26 & 0,65 & 0,36 & & & FB027 & 0,59 & 0,41 & & \\
\hline FB028 & 0,53 & 0,52 & & 0,32 & FB029 & 0,45 & 0,56 & & \\
\hline FB030 & 0,67 & 0,36 & & & FB031 & 0,59 & 0,39 & & \\
\hline FB032 & 0,57 & 0,36 & & 0,37 & FB033 & 0,54 & 0,45 & & \\
\hline FB034 & 0,56 & 0,51 & & & FB035 & 0,47 & 0,62 & & \\
\hline FB036 & 0,66 & 0,44 & & & FB037 & 0,61 & 0,47 & & \\
\hline FBO42 & & & & $-0,61$ & $\mathrm{FBO43}$ & & & & $-0,68$ \\
\hline FBO44 & $-0,33$ & & & $-0,71$ & FBO45 & $-0,37$ & & & $-0,72$ \\
\hline FBO46 & $-0,37$ & & $-0,31$ & $-0,68$ & $\mathrm{FBO47}$ & $-0,36$ & & & $-0,71$ \\
\hline FB050 & & & 0,63 & & FB051 & & & 0,59 & \\
\hline FB052 & & & 0,57 & & FB053 & & & 0,52 & \\
\hline FB058 & & & 0,58 & & FB059 & & & 0,63 & \\
\hline FB061 & $-0,30$ & & $-0,55$ & & FB062 & $-0,39$ & & $-0,47$ & \\
\hline FB063 & & & $-0,68$ & & FB064 & & & $-0,58$ & \\
\hline FB0106 & 0,42 & 0,61 & & & FB0107 & & 0,59 & 0,31 & \\
\hline FB0108 & 0,45 & 0,53 & & & FB0109 & & 0,58 & & \\
\hline FB0110 & & 0,67 & & & FB0111 & & 0,65 & & \\
\hline FB0112 & & & 0,71 & & FB0113 & & & 0,70 & \\
\hline FB0114 & & 0,31 & 0,59 & & FB0115 & & & 0,67 & \\
\hline FB0116 & & 0,69 & & & FB0117 & & 0,65 & 0,30 & \\
\hline FB0118 & & 0,60 & 0,36 & & FB0119 & & 0,65 & 0,31 & \\
\hline FB0120 & 0,30 & 0,64 & & 0,32 & FB0121 & 0,33 & 0,61 & & 0,37 \\
\hline FB0122 & & 0,67 & & 0,34 & FB0123 & & 0,62 & & 0,37 \\
\hline FB0126 & 0,35 & 0,54 & & & FB0127 & 0,37 & 0,54 & & \\
\hline FB0128 & 0,32 & 0,64 & & & FB0129 & 0,36 & 0,57 & & \\
\hline FB0136 & & & & $-0,63$ & FB0137 & & & & $-0,65$ \\
\hline FB0138 & & & & $-0,60$ & FB0139 & & & & $-0,60$ \\
\hline
\end{tabular}


atingia valores de saturação no limite de ,30 ou abaixo deste. Com a análise do scree plot, percebeu-se que os dados eram compatíveis com a escolha de quatro factores. Com uma nova análise factorial forçada a quatro factores, os itens 39, 41, 49, 55 e 131 foram eliminados (em função de os loadings não atingirem os critérios definidos). Apesar de alguns itens saturarem em mais do que um factor, optámos pelo maior valor de loading e/ou pelo factor que melhor justificava a inclusão do item, ficando o Questionário de Cuidados Maternos constituído também por 37 itens (tal como o do Pai). O valor de Kaiser-Meyer-Olkin foi de ,933, o qual é considerado excelente ${ }^{21}$, e o teste de esfericidade de Bartlett é de $\mathrm{X}^{2}=5859,03$, o qual atinge um valor significativo $(\mathrm{p}<, 05)$, pelo que se rejeita a hipótese nula e confirma-se que as variáveis são correlacionáveis. A análise factorial mostra que $37,30 \%$ da variância é explicada pelo factor $1,7,08 \%$, pelo factor 2, 4,69\%, pelo factor 3 e $4,30 \%$, pelo factor 4. Em termos globais, $53,37 \%$ da variância é explicada pelos quatro factores, cujo resultado está dentro dos limites científicos aceitáveis ${ }^{21}$. Para verificar a forma como os itens se distribuem pelos quatro factores, consulte a tabela 1.

\section{c) Questionário do Ambiente Familiar}

A análise factorial inicialmente realizada com rotação varimax dos 81 itens deste questionário sem forçar nenhum factor distribui os itens por 23 factores. No entanto, sua distribuição não se agrupava em torno de nenhum constructo teórico bem definido e atingia valores de saturação no limite de ,30 ou abaixo deste. Com a análise do scree plot, percebeu-se que os dados eram compatíveis com a escolha de seis factores. Com uma nova análise factorial forçada a seis factores, os itens $68,71,73,74,75,78,79,81,91,94,96,97,101$, $105,134,135,153,169,170,171,172,173,174,175,176,177,178$ e 179 foram eliminados (em decorrência de os loadings não atingirem os critérios definidos), ficando o Questionário do Ambiente Familiar constituído por 50 itens. O valor de Kaiser-Meyer-Olkin foi de ,765, o qual é considerado razoável ou médio ${ }^{21}$, e o teste de esfericidade de Bartlett é de $\mathrm{X}^{2}=9467,56$, o qual atinge um valor significativo $(\mathrm{p}<, 05)$, pelo que se rejeita a hipótese nula e confirma-se que as variáveis são correlacionáveis. A análise factorial mostra que 18,61\% da variância é explicada pelo factor $1,9,54 \%$, pelo factor $2,7,86 \%$, pelo factor $3,6,31 \%$, pelo factor $4,4,85 \%$, pelo factor 5 e $4,14 \%$, pelo factor 6 . Na sua globalidade, os seis factores explicam $51,31 \%$ da variância, cujo resultado está dentro dos limites científicos aceitáveis ${ }^{21}$. Para verificar a forma como os itens se distribuem pelos seis factores, consulte a tabela 2 .

Como a cotação dos itens sugere que quanto mais próximo de 5 melhor é o cuidado e/ou ambiente familiar, a cotação de vários itens foi invertida e recodificada, apoiada quer nos resultados da análise factorial (loadings negativos conforme tabela 1), quer da análise do conteúdo semântico de cada um. Assim, no Questionário de Cuidados Paternos, a cotação dos itens 42, 44, 46, 61, 63, 136 e 138 foi invertida.

De acordo com os dados disponíveis para o Questionário dos Cuidados Paternos na tabela 1, considerando o conteúdo teórico dos itens, o factor 1, constituído por 16 itens, foi caracterizado por "Aceitação e Expressão de Afecto Paterno". O factor 2 refere-se à "Responsividade e Diálogo Paterno" e é constituído por 9 itens. O factor 3 é "Envolvimento Educacional Paterno" e é constituído por 7 itens. O factor 4 é "Respeito Físico e Emocional Paterno" e é constituído por 5 itens.

No Questionário de Cuidados Maternos, a cotação dos itens 43, $45,47,62,64,137$ e 139 foi também invertida. Na mesma tabela 1, verificámos que a organização obtida para o Questionário dos Cuidados Maternos é equivalente à organização obtida para o Questionário dos Cuidados Paternos, o que facilita uma comparação posterior entre os pais. Assim sendo, o factor 1 denomina-se "Aceitação e Expressão de Afecto Materno", o factor 2, "Responsividade e Diálogo Materno", o factor 3, "Envolvimento Educacional Materno" e o factor 4, "Respeito Físico e Emocional Materno".
Tabela 2. Loadings do Questionário do Ambiente Familiar na análise factorial forçada a seis factores

\begin{tabular}{|c|c|c|c|c|c|c|}
\hline Item & Factor 1 & Factor 2 & Factor 3 & Factor 4 & Factor 5 & Factor 6 \\
\hline FB013 & & & $-0,59$ & & & \\
\hline FB014 & & & $-0,66$ & & & \\
\hline FB015 & & & $-0,63$ & & & \\
\hline FB065 & & & & & 0,65 & \\
\hline FB066 & & & & & 0,65 & \\
\hline FB069 & & & & & 0,70 & \\
\hline FB070 & & & & & 0,70 & \\
\hline FB072 & & & & & & 0,46 \\
\hline FB076 & & & & & & 0,57 \\
\hline FB077 & & & & & & 0,58 \\
\hline FB080 & & & & & & 0,55 \\
\hline FB082 & & & & & & 0,58 \\
\hline FB083 & & & & & & 0,57 \\
\hline FB084 & & & & 0,71 & & \\
\hline FB085 & & & & 0,84 & & \\
\hline FB086 & & & & 0,85 & & \\
\hline FB087 & & & & 0,83 & & \\
\hline FB088 & & & & 0,56 & & \\
\hline FB089 & & & & 0,73 & & \\
\hline FB090 & & & & 0,57 & & \\
\hline FB092 & & 0,30 & & & & $-0,45$ \\
\hline FB093 & & & & & & $-0,48$ \\
\hline FB098 & & 0,58 & & & & \\
\hline FB099 & & 0,71 & & & & \\
\hline FB0100 & & 0,69 & & & & \\
\hline FB0102 & & & & & 0,60 & \\
\hline FB0103 & & $-0,34$ & & & 0,57 & \\
\hline FB0104 & & & & & 0,48 & \\
\hline FB0132 & & 0,56 & & & & \\
\hline FB0133 & 0,43 & 0,32 & & & & \\
\hline FB0140 & & 0,43 & 0,39 & & & \\
\hline FB0141 & & 0,35 & 0,53 & & & \\
\hline FB0142 & & 0,42 & 0,55 & & & \\
\hline FB0143 & 0,35 & & 0,65 & & & \\
\hline FB0144 & & 0,37 & 0,56 & & & \\
\hline FB0145 & 0,37 & & 0,65 & & & \\
\hline FB0155 & 0,75 & & & & & \\
\hline FB0156 & 0,76 & & 0,41 & & & \\
\hline FB0157 & 0,77 & & & & & \\
\hline FB0158 & 0,72 & & 0,48 & & & \\
\hline FB0159 & 0,79 & & & & & \\
\hline FB0160 & 0,71 & & 0,42 & & & \\
\hline FB0161 & 0,70 & & & & & \\
\hline FB0162 & 0,61 & & 0,36 & & & \\
\hline FB0163 & & 0,78 & & & & \\
\hline FB0164 & & 0,76 & & & & \\
\hline FB0165 & & 0,65 & & & & \\
\hline FB0166 & & 0,66 & & & & \\
\hline FB0167 & 0,54 & 0,39 & & & & \\
\hline FB0168 & 0,45 & 0,42 & & & & \\
\hline
\end{tabular}


No Questionário do Ambiente Familiar e de acordo com a tabela 2, o factor 1 foi denominado "Ausência de Abuso de Substâncias Parental", o factor 2, "Coligação Parental e Ajustamento Psicológico Parental", o factor 3, "Cuidado Físico e Relacionamento Parental Não Violento", o factor 4, "Ausência de Tarefas Domésticas", o factor 5, "Apoio Social Infantil e Expressão Emocional Familiar", e o factor 6, "Controlo Parental". Considerando sempre o sentido em que 5 significa melhor ambiente, todos os itens do factor 1 nessa escala foram invertidos (para que, quanto mais para 5, menor abuso de substâncias parental), assim como os do factor 2 (para que, quanto mais para 5, maior coligação e ajustamento psicológico parental). No factor 6 , os itens $72,76,77,80,82$ e 83 foram recodificados $(1=1,2$ $=2,3=5,4=4,5=3$ ) e 92 e 93 foram invertidos (para que, quanto mais próximo de 5 , melhor e mais democrático e decisões em família no Controlo Parental). No factor 3, os itens 140, 141, 142, 143, 144 e 145 sobre violência doméstica foram invertidos (para que, quanto mais para 5, menos violência, mantendo-se, portanto, os itens 13 , 14 e 15 de 1 a 5$)$.

\section{Consistência interna}

Foi estabelecido como critério mínimo nas correlações item-total para cada subescala de ,40 e de ,30 nos valores de squared multiple correlation, desde que a eliminação desse item não baixasse o valor de alfa de Chronbach do factor e não prejudicasse a relação com seu questionário constituinte (para que comparações entre pai e mãe pudessem ser concretizadas).

\section{a) Questionário de Cuidados Paternos}

O alfa de Cronbach total do Questionário de Cuidados Paternos é de ,954. A análise dos coeficientes dos alfas de Cronbach, considerando a organização dos itens pelas 4 subescalas, varia desde, 739 (escala 4 - Respeito Físico e Emocional Paterno) até ,946 (escala 1 - Aceitação e Expressão de Afecto Paterno).

\section{b) Questionário de Cuidados Maternos}

O alfa de Cronbach total do Questionário de Cuidados Maternos é de ,945. A análise dos coeficientes dos alfas de Cronbach, considerando a organização dos itens pelas 4 subescalas, varia desde,743 (escala 3
- Envolvimento Educacional Materno) até,938 (escala 1 - Aceitação e Expressão de Afecto Materno).

\section{c) Questionário do Ambiente Familiar}

O alfa de Cronbach total do Questionário do Ambiente Familiar é de ,843. A análise dos coeficientes dos alfas de Cronbach, considerando a organização dos itens pelas 6 subescalas, varia desde, 739 (escala 6 - Controlo Parental) até ,888 (escala 1 - Abuso de Substâncias Parental).

Analisada a consistência interna de cada questionário, procedeuse ao cálculo das médias e desvios-padrões da amostra analisada, cujos valores estão expostos na tabela 3 . Atendendo ao valor de cada uma das subescalas constituintes de cada questionário, são calculadas quatro novas variáveis - Índice de Cuidados Paternos, Índice de Cuidados Maternos, Índice de Ambiente Familiar e Índice Geral de Cuidados Familiares. Essas variáveis, que permitem um valor global das memórias do indivíduo em relação às suas experiências na família de origem (com o pai, mãe, do ambiente familiar e dos cuidados familiares em geral), surgem da média do somatório do valor com que cada sujeito classificou as suas respostas em cada uma das subescalas de cada questionário, pelo que o valor final varia entre 1 e 5 (ver Tabela 3 ).

Por meio do teste de Kolmogorov-Smirnov, verificamos que a distribuição na nossa amostra de todos os questionários é não normal, excepto para as subescalas "Responsividade e Diálogo Paterno" e "Responsividade e Diálogo Materno" ( $p>$,05). Assim, foi realizado um Mann-Whitney para verificar se havia diferenças entre o sexo masculino e feminino. Perante esses resultados, constatámos que existem diferenças estatisticamente significativas nas subescalas "Apoio Social e Expressão Emocional Familiar" ( $U=5629,50$, $Z=-6,15, p<, 001)$ e "Controlo Parental" $(U=7261,50, Z=-3,74$, $\mathrm{p}<, 001$ ), com os homens a evidenciar valores mais elevados de interacção e apoio social infantil, e expressão emocional familiar e menos controlo parental comparativamente com as mulheres. Daqui se constata que, relativamente ao "Controlo Parental", considerando que esse varia entre um continuum de 1 (autocrático, com decisão dos pais sem opinião do filho), passando por 3 (decisão autónoma e individual, sem os pais) até 5 (decisão democrática, conjunta em família), as mulheres têm uma percepção de um tipo de controlo mais democrático $(\mathrm{M}=3,39, \mathrm{DP}=, 74)$, comparativamente com os homens $(\mathrm{M}=3,07, \mathrm{DP}=, 62)$ - que estão mais próximos de valores

Tabela 3. Médias e desvios-padrões das subescalas dos Questionários dos Cuidados Familiares

\begin{tabular}{|c|c|c|c|c|c|c|}
\hline \multirow[t]{2}{*}{ Nome da escala } & \multicolumn{2}{|c|}{$\begin{array}{c}\text { Total } \\
(\mathrm{N}=280)\end{array}$} & \multicolumn{2}{|c|}{$\begin{array}{l}\text { Homens } \\
(\mathrm{N}=135)\end{array}$} & \multicolumn{2}{|c|}{$\begin{array}{l}\text { Mulheres } \\
(\mathrm{N}=145)\end{array}$} \\
\hline & Média & $\mathrm{DP}$ & Média & $\mathrm{DP}$ & Média & $\mathrm{DP}$ \\
\hline 1 - Aceitação e Expressão de Afecto Paterno & 3,97 & 837 & 3,98 & 832 & 3,96 & .844 \\
\hline 2 - Responsividade e Diálogo Paterno & 3,46 & 897 & 3,53 & 808 & 3,40 & 971 \\
\hline 3 - Envolvimento Educacional Paterno & 3,70 & 784 & 3,69 & ,744 & 3,72 & 823 \\
\hline 4 - Respeito Físico e Emocional Paterno & 4,19 & 762 & 4,13 & ,731 & 4,23 & ,790 \\
\hline ÍNDICE DE CUIDADOS PATERNOS & 3,831 &, 660 & 3,83 &, 610 & 3,83 & ,706 \\
\hline 1 - Aceitação e Expressão de Afecto Materno & 4,25 & 697 & 4,30 & 674 & 4,20 & ,717 \\
\hline 2 - Responsividade e Diálogo Materno & 3,76 & 769 & 3,77 & .702 & 3,75 &, 829 \\
\hline 3 - Envolvimento Educacional Materno & 3,95 & 682 & 3,96 & 643 & 3,94 & ,718 \\
\hline 4 - Respeito Físico e Emocional Materno & 4,19 & 802 & 4,20 & .779 & 4,18 & .825 \\
\hline ÍNDICE DE CUIDADOS MATERNOS & 4,038 &, 576 & 4,05 &, 529 & 4,02 &, 617 \\
\hline 1 - Ausência de Abuso de Substâncias Parental & 4,91 & 382 & 4,89 & .461 & 4,92 & ,290 \\
\hline 2 - Coligação/Ajustamento Psicológico Parental & 4,01 & .611 & 4,07 &, 598 & 3,94 &, 618 \\
\hline 3 - Negligência Física e Violência Doméstica & 4,75 & .455 & 4,74 & .513 & 4,75 & ,395 \\
\hline 4 - Tarefas Domésticas & 2,82 & 1,251 & 2,33 & 1,020 & 3,28 & 1,276 \\
\hline 5-Apoio Social e Expressão Emocional Familiar & 3,61 & .764 & 3,60 & .719 & 3,63 & .805 \\
\hline 6 - Controlo Parental & 4,03 & .499 & 4,15 & .466 & 3,91 &, 503 \\
\hline ÍNDICE AMBIENTE FAMILIAR & 4,02 & 321 & 3,96 & ,307 & 4,07 & ,327 \\
\hline ÍNDICE GERAL DE CUIDADOS FAMILIARES & 3,972 &, 404 & 3,95 & 390 & 3,99 & ,418 \\
\hline
\end{tabular}


mais autónomos e individuais. Nas escalas restantes, não se verificaram diferenças estatisticamente significativas $(\mathrm{p}>, 05)$.

\section{Relação entre cuidados paternos, maternos e ambiente familiar}

Fez-se uma análise correlacional (ver Tabela 4), com recurso à correlação de Spearman entre os diferentes factores e índices do instrumento, cujos resultados mostram que sua quase totalidade está, entre si, significativa e positivamente inter-correlacionada $(\mathrm{p}<, 05)$. Verificámos, no entanto, duas correlações negativas estatisticamente significativas, apesar de baixas, entre a "ausência de tarefas domésticas" com a "coligação e ajustamento psicológico parental" ( $r s=$ - ,139, $\mathrm{p}<, 05)$ e com o "controlo parental" ( $\mathrm{rs}=-, 224, \mathrm{p}<, 01$ ).

Em suma, a avaliação das experiências familiares utilizando os 3 questionários (124 itens), mais os 5 itens de abuso sexual enquanto checklist, envolve responder a 129 itens. Nesta checklist, as respostas são classificadas em termos de "Sim, houve abuso" ou "Não houve abuso". Para os nossos participantes, $0,4 \%(\mathrm{~N}=1)$ relata que foi abusado pelo pai, $0,4 \%(\mathrm{~N}=1)$ relata que foi abusado pela mãe, $1,8 \%$ $(\mathrm{N}=5)$ relata que foi abusado por algum familiar (por exemplo, irmão, tio, primo etc.), $1,1 \%(\mathrm{~N}=3)$ foi abusado com o uso de força física por pessoas fora do núcleo familiar (por exemplo, vizinho, ama, professor, desconhecido etc.) e $2,5 \%(\mathrm{~N}=7)$ foram abusados por alguém fora do núcleo familiar sem o uso da força física.

Em termos globais, o total dos três questionários deu origem a um índice de experiências familiares, cujo valor médio total é de $3,94(\mathrm{DP}=, 74)$.

\section{Discussão}

Este estudo, que parte de um instrumento muito extenso sobre os cuidados recebidos durante o desenvolvimento na família de origem, avalia as qualidades psicométricas de três questionários que totalizam 124 itens - Questionário de Cuidados Paternos (37 itens), Questionário de Cuidados Maternos (37 itens) e Questionário do Ambiente Familiar (50 itens) - que procuram averiguar a forma como os jovens adultos (maiores de 18 anos) relatam dimensões do funcionamento familiar. Considerando as recomendações da European Federation of Psychological Associations ${ }^{22}$, importa salientar que as áreas de aplicação desses materiais de auto-resposta são transversais aos vários campos da psicologia, mas está mais indicado para a área da clínica do adulto (individual ou familiar), com uma administração tanto individual como colectiva.

Na presente investigação, os factores constituintes de cada um dos questionários demonstram uma validade factorial e consistência interna adequadas. O estudo das qualidades psicométricas de cada subescala dentro de cada um dos três questionários evidenciou valores de alfa elevados, que variam entre ,74 e ,95. O estudo das correlações entre as várias subescalas evidencia valores que se apresentam, na maior parte das vezes, com magnitude elevada e significativa, sem ultrapassar o valor de ,90 (o que iria estar a avaliar a mesma coisa) ${ }^{22}$. Nesse sentido, verificámos que muitas das correlações são positivas entre os factores dos três questionários, excepto no que diz respeito às subescalas de "apoio social e expressão emocional familiar" e "controlo parental". A análise específica das correlações positivas mais robustas indica que, quando os indivíduos relatam que experienciavam responsividade e diálogo com os pais, tendiam a sentir maior aceitação e expressão de afectos dos pais. Também se constata que, quando os indivíduos experienciavam respeito físico e emocional da mãe e envolvimento educacional da mãe, tendiam a sentir o mesmo por parte do pai. Na correlação negativa verificada no apoio social e expressão emocional familiar e tipo de controlo parental, apesar do valor baixo desta, e considerando as hipóteses de resposta no "controlo parental", os resultados da nossa amostra revelam que a correlação negativa pode significar que, quanto mais os participantes sentiam os pais como democráticos (com menos uso do seu poder), menos apoio social e expressão emocional familiar sentiam. Achamos pouco esperada essa relação, pois seria de esperar uma correlação positiva também, pelo que mais estudos são necessários, com amostras maiores, para analisar a relação a essas escalas.

A divisão dos itens pelos três questionários parece-nos mais favorável do que um único instrumento por inúmeras razões, a saber: 1) não há sobreposição dos itens nas diferentes escalas ou factores (ao contrário da versão original); 2) em função da análise factorial, a diminuição do número de itens também se revelou positiva, uma vez que se simplificaram os questionários; 3 ) com a redução do número de itens e a organização de três questionários, o tempo de aplicação tornou-se menor; 4) aumenta a flexibilidade do clínico ou do investigador, pois permite escolher qual ou quais os questionários a aplicar, pois nem todos os indivíduos tiveram ou viveram com ambos os pais (devido a óbito, divórcio ou ausência). Com a divisão em três questionários, respondem apenas àquele com quem habitam. Dessa forma, alivia-se também a carga emocional de não poder responder nos vários itens acerca da pessoa ausente; 5) ao separar os questionários, evita o contraste que poderá existir na relação com o pai e a mãe e o não querer favorecer ou denegrir uma figura parental em função da outra ao longo das respostas ao questionário.

A análise de diferenças entre homens e mulheres revelou que as mulheres relatam maior "respeito físico e emocional do pai" e uma maior "coligação e ajustamento psicológico parental (em termos de ambiente familiar) do que os homens. Acresce ainda o facto de que os homens têm menor ausência de tarefas domésticas e um controlo

Tabela 4. Matriz de correlações de Spearman das 14 subescalas da versão portuguesa dos 3 questionários

\begin{tabular}{c|c|c|c|c|c|c|c|c|c|c|c|c|c|c}
\hline & 1 & 2 & 3 & 4 & 5 & 6 & 7 & 8 & 9 & 10 & 11 & 12 & 13 & 14 \\
\hline 1 & 1 & & & & & & & & & & & & & \\
\hline 2 &, $772^{* *}$ & 1 & & & & & & & & & & & \\
\hline 3 &, $486^{* *}$ &, $454^{* *}$ & 1 & & & & & & & & & & \\
\hline 4 &, $411^{* *}$ &, $424^{* *}$ &, $135^{*}$ & 1 & & & & & & & & & \\
\hline 5 &, $680^{* *}$ &, $517^{* *}$ &, $362^{* *}$ &, $241^{* *}$ & 1 & & & & & & & & \\
\hline 6 &, $519^{* *}$ &, $693^{* *}$ &, $338^{* *}$ &, $265^{* *}$ &, $758^{* *}$ & 1 & & & & & & & \\
\hline 7 &, $249^{* *}$ &, $224^{* *}$ &, $651^{* *}$ &,- 046 &, $422^{* *}$ &, $378^{* *}$ & 1 & & & & & & \\
\hline 8 &, $330^{* *}$ &, $324^{* *}$ &, $153^{*}$ &, $761^{* *}$ &, $390^{* *}$ &, $373^{* *}$ &, 089 & 1 & & & & & \\
\hline 9 &, 054 &, 027 &, 074 &,- 054 &, 088 &, 042 &,- 010 &, 054 & 1 & & & & \\
\hline 10 &, $423^{* *}$ &, $363^{* *}$ &, $272^{* *}$ &, $306^{* *}$ &, $224^{* *}$ &, $199^{* *}$ &, 071 &, $253^{* *}$ &, $222^{* *}$ & 1 & & & \\
\hline 11 &, $452^{* *}$ &, $386^{* *}$ &, $403^{* *}$ &, $306^{* *}$ &, $407^{* *}$ &, $347^{* *}$ &, $254^{* *}$ &, $308^{* *}$ &, $214^{* *}$ &, $347^{* *}$ & 1 & & \\
\hline 12 &,- 105 &,- 101 &, 036 &,- 041 &,- 051 &,- 034 &, 054 &,- 060 &, 037 &,$- 139^{*}$ &,- 103 & 1 & \\
\hline 13 &, $427^{* *}$ &, $438^{* *}$ &, $267^{* *}$ &, 114 &, $379^{* *}$ &, $369^{* *}$ &, $283^{* *}$ &, $120^{*}$ &, 022 &, 099 &, $173^{* *}$ &,- 086 & 1 & \\
\hline 14 &, 080 &, 097 &,- 057 &, $164^{* *}$ &, 082 &, 051 &,- 010 &, $137^{*}$ &, 073 &, 111 &, $147^{*}$ &,$- 224^{* *}$ &, $142^{*}$ & 1 \\
\hline
\end{tabular}

${ }^{*} p<, 05 .{ }^{* *} p<, 01$. 
parental mais democrático e com maior liberdade para as suas tomadas de decisão do que as mulheres.

Relativamente às limitações deste estudo, a falta de uma análise directa da veracidade das respostas enfraquece a confiança que é depositada nos resultados obtidos. A auto-resposta ao questionário associada à informação recolhida doutra forma (no exterior com os familiares e/ou outros registos) teria complementado e ajudado a validar os resultados. Apesar de difícil e muitas vezes impossível, informação adicional dos membros familiares, médicos ou legais ajudaria a fortalecer a fiabilidade das variáveis usadas neste estudo e poderia ser usada para examinar a validade dos resultados para cada factor das escalas analisadas ${ }^{23-25}$. A recordação é uma actividade basicamente reconstrutiva que assenta num dinamismo constante e transitório, relacionada não só com as representações mnésicas internas, mas também com as características da situação em que se recorda ${ }^{23,26}$. As investigações retrospectivas têm três limitações metodológicas, pois comportam alguns riscos no que se refere à interpretação dos dados, uma vez que pode levar a confusões quanto à rigorosa demarcação entre causas e consequências das EAI (por exemplo, a alteração do comportamento da criança pode desencadear episódios de maus-tratos ou pode ser sua consequência) ${ }^{1,16,17,24}$. Numa segunda limitação, pode ser pelo facto de se estar a investigar com casos provenientes dos serviços de protecção infantil, o que limita a generalização dos resultados. E, por último, dadas as dificuldades conceptuais na definição do maltrato, ainda existem muitas investigações que tratam esse conceito como global, quando, na sua essência, apenas foram trabalhados com casos de abuso físico ou outro tipo de abuso. Podemos também salientar como limitação o facto de o instrumento avaliar um longo período de desenvolvimento (infância e adolescência), o que torna mais difícil determinar se, ao responder às questões, os sujeitos estão a pensar na mesma altura da vida. Assim, estudos comparativos com outros instrumentos ou mais restritos em relação ao momento de vida que estão a tentar avaliar são necessários em futuras investigações.

Uma outra limitação deste estudo é o facto de a amostra que serviu de base para sua realização não ser representativa da população portuguesa, dado que são jovens adultos e estudantes. Assim, apesar de os resultados dessa investigação serem encorajadores, sugere-se que, em estudos futuros, esse estudo seja replicado com uma amostra representativa da população portuguesa. Aliás, apesar dos indicadores positivos da utilidade desses questionários, falta ainda estudar sua validade e reprodutibilidade. Em futuros estudos, é importante avaliar a fiabilidade teste-reteste para os três questionários e também aumentar o número de participantes e variabilidade das suas características etárias e sócio-económicas por forma a garantir uma maior probabilidade de generalização dos resultados para a população portuguesa.

\section{Agradecimentos}

Aos Professores Doutores Timothy Melchert (Marquette University) e Thomas Sayger (The University of Memphis), pelo apoio prestado e opiniões.

\section{Referências}

1. Melchert T. The Development of the Family Background Questionnaire. Non-published Doctorate's Dissertation. 1991. Memphis: University of Memphis.

2. Golder S, Gillmore M, Spieker S, Morrison D. Substance use, related problem behaviours and adult attachment in a sample of high risk older adolescent women. J Child Fam Stud. 2005;14:181-93.
3. Rosenman S, Rodgers B. Childhood adversity in an Australian population. Soc Psychiatry Psychiatr Epidemiol. 2004;39:695-702.

4. Sternberg K, Lamb M, Gutermann E, Abbott C. Effects of early and later family violence on children's behavior problems and depression: a longitudinal, multi-informant perspective. Child Abuse Negl. 2006;30:283-306.

5. Cardoso RM. Auto-regulação dos sistemas naturais. Texto pormenorizado da lição síntese, para candidatura ao título de agregado. Faculdade de Medicina da Universidade do Porto; 1990. Porto: Material não publicado.

6. Felitti VJ. The relationship of adverse childhood experiences to adult health: turning gold into lead. Permanente Journal. 2002;6(1):44-7.

7. Anda RF, Whitfield CL, Felitti VJ, Chapman D, Edwards VJ, Dube SR, et al. Adverse childhood experiences, alcoholic parents, and later risk of alcoholism and depression. Psychiatr Serv. 2002;53(8):1001-9.

8. Edwars VJ, Holden GW, Felitti VJ, Anda RF. Relationship between multiple forms of childhood maltreatment and adult mental health in community respondents: results from the Adverse Childhood Experiences Study. Am J Psychiatry. 2003;160(8):1453-60.

9. Bifulco A, Moran P. Wednesday child: research into women's experience of neglect and abuse in childhood and adult depression. London: Routledge; 1998.

10. Johnson JG, Cohen P, Brown J, Smailes EM, Berstein DP. Childhood maltreatment increases risk for personality disorders during early adulthood. Arch Gen Psychiatry. 1999;56(7):600-6.

11. Ainsworth MDS, Blehar MC, Waters Z, Wall S. Patterns of Attachment - a psychopathological study of the strange situation. Hillsdale, NJ: Lawrence Erlbaum Associates; 1978

12. Bowlby J. A secure base: parent-child attachment and health human development. New York: Basic Books; 1988.

13. Melchert T, Sayer T. The development of an instrument for measuring memories of family of origin characteristics. Educ Psychol Meas. 1998;58(1):99-118.

14. Malinosky-Rummell R, Hansen DJ. Long-term consequences of childhood physical abuse. Psychol Bull. 1993;114:68-79.

15. Azevedo MC, Maia AC. Maus-tratos à criança. Lisboa: Climepsi Editores; 2006.

16. Parker G, Tupling H, Brown LB. A parental bonding instrument. Br J Med Psychol. 1979;52:1-10.

17. Melchert $\mathrm{T}$. Testing the validity of an instrument for assessing family of origin history. J Clin Psychol. 1998;54(7):863-75.

18. Monteiro IS, Maia A. Understanding depression: the contribution of the family background. Poster session presented at the 9th European Congress of Psychology, Granada: Spain; 2005.

19. Spielberger C, Sharma S. Cross-cultural measurement of anxiety. In: Spielberger C, Diaz-Guerrero R, eds. Cross-cultural anxiety. New York: John Wiley \& Sons; 1976. p. 13-25.

20. Van de Vijver F, Hambleton R. Translating tests: some practical guidelines. European Psychologist. 1996;1(2):89-99.

21. Pett MA, Lackey NR, Sullivan JJ. Making sense of factor analysis: the use of factor analysis for instrument development in health care research. 2003. Sage Publications, Inc.

22. EFPA - European Federation of Psychological Associations. EFPA Review Model for the description and evaluation of psychological tests (updated and revised). 2005. Version 3.4r de 8/11/2005.

23. Williams JMG, Watts F, Macleod C, Mathews A. Psicologia cognitiva e perturbações emocionais. Lisboa: Climepsi; 2000.

24. Férnandez A, Díez E. Memoria y distorsión. In: Sanchez Cabaco A, Beato MS, eds. Psicología de la memoria: ámbitos aplicados. Madrid: Alianza; 2001. p.159-70.

25. Pergher GK, Stein LM, Wainer R. Estudos sobre a memória na depressão: achados e implicações para a terapia cognitiva. J Bras Psiquiatr. 2004;31(2):82-90.

26. Silva Filho ACP. Terapia cognitiva construtivista e psicanálise. Rev Psiq Clín. 2002;29(3):164-6. 